\title{
Accumulation and hoar effects on microwave emission in the Greenland ice-sheet dry-snow zones
}

\author{
Waleed Abdalati, ${ }^{1}$ Konrad Steffen ${ }^{2}$ \\ ${ }^{1}$ Universities Space Research Association, NASA Goddard Space Flight Center, Greenbelt, Maryland 20771, U.S.A. \\ ${ }^{2}$ Center for the Study of Earth from Space, Cooperative Institute for Research in Environmental Sciences, University of Colorado, \\ Boulder, Colorado 80309, U.S.A.
}

\begin{abstract}
The relationships between accumulation and microwave emissivity in the dry-snow regions of the Greenland ice sheet are studied with special focus on hoar formation. With the use of a discrete-ordinate radiative-transfer model, the relative contributions of hoar and accumulation to microwave emission are examined. The results show that the link between microwave emission and accumulation rates in the dry-snow area of the Greenland ice sheet is significant; however, the emissivity is also dependent on the extent of hoar development. As a result, accumulation estimates based on passive microwave observations in the low-accumulation dry-snow areas will require successful parameterization of hoar formation. Furthermore, the increasing $T_{\mathrm{b}}$ trends that were observed during the 1980 s, which have been hypothesized to be related to accumulation changes, may be strongly related to variations in hoar development. Other possible explanations for these observed trends, such as slight surface melt or atmospheric conditions, are also discussed.
\end{abstract}

\section{INTRODUCTION}

The Greenland ice sheet (Fig. 1) plays a major role in Arctic and global climates by acting as a topographic barrier to large-scale air-mass flow and also through its exchange of energy, moisture and momentum with the atmosphere. Through its fecd-back mechanisms, the ice sheet both contributes and responds to fluctuations in the regional climate (Dickenson and others, 1987). As a result, understanding the behavior of this vast expanse of ice in the changing climate is of great interest.

The current state of mass balance of the Greenland ice sheet and its variation with climate are not well understood. Surface melt has shown signs of a significant increase in recent years (Mote and Anderson, 1995; Abdalati and Steffen, 1997) and ice-sheet thickness in parts of Greenland south of $72^{\circ} \mathrm{N}$ has also been observed to increase (Zwally and others, 1989). However, little has been done to assess the accumulation rates in the northern parts of the ice sheet. It has been suggested that an accumulation signal in these northern areas may be apparent in the satellite microwave brightness temperatures of the Scanning Multi-channel Microwave Radiometer (SMMR) and the Special Sensing Microwave Imager (SSM/I) (Steffen and others, 1993).

A direct relationship between snow accumulation and microwave emissivity $(\varepsilon)$ in the dry-snow regions of the Greenland and Antarctic ice sheets has been shown (Zwally, 1977; Comiso and others, 1982; Zwally and Giovinetto, 1995). This relationship is manifested in the microwave brightness temperature $\left(T_{\mathrm{b}}\right)$ according to the RayleighJeans approximation:

$$
T_{\mathrm{b}}=\varepsilon T_{\mathrm{p}}
$$

where $T_{\mathrm{p}}$ is the effective physical temperature of the emitting snow and is weighted by a radiative-transfer function.

Significant changes in brightness temperature were observed during the SMMR coverage period in the drysnow regions of the ice sheet (Steffen and others, 1993). In some instances, the average $T_{\mathrm{b}}$ increased by $18 \mathrm{~K}$ in 6 years (Fig. 2) with the strongest trends located in the northeast part of the ice sheet, near Tunu and Inge-Lehman (Fig. 1), where accumulation rates are lowest (Ohmura and Reeh, 1991). It has been hypothesized that these trends may be attributable to interannual variations in accumulation rates and associated changes in volume scattering and emissivity, since they are so large and apparently not linked to temperature changes (Steffen and others, 1993). The relationship between $T_{\mathrm{b}}$ and accumulation, however, is complicated by the development of hoar layers within and at the surface of the snowpack. These layers strongly affect the overall microwave emission.

In this study, we revisit the relationships between accumulation and microwave emissivity with the additional consideration of hoar formation. The relative impacts of each on the microwave emission are examined using a radiative-transfer model. The primary objective is to assess the importance of hoar development in the detection of snow accumulation from observed brightness temperatures, and the results are expected to aid in the interpretation of how temporal changes in microwave brightness temperatures relate to changes in accumulation rate. We also hope to understand better the increase in $T_{\mathrm{b}}$ observed in the SMMR record. This analysis focuses on microwave emissivities rather than brightness temperatures, in order to eliminate the need for detailed knowledge of the temperature structure in the firn and atmospheric conditions. Such knowledge would be required to calculate $T_{\mathrm{b}} \mathrm{s}$; however, for 


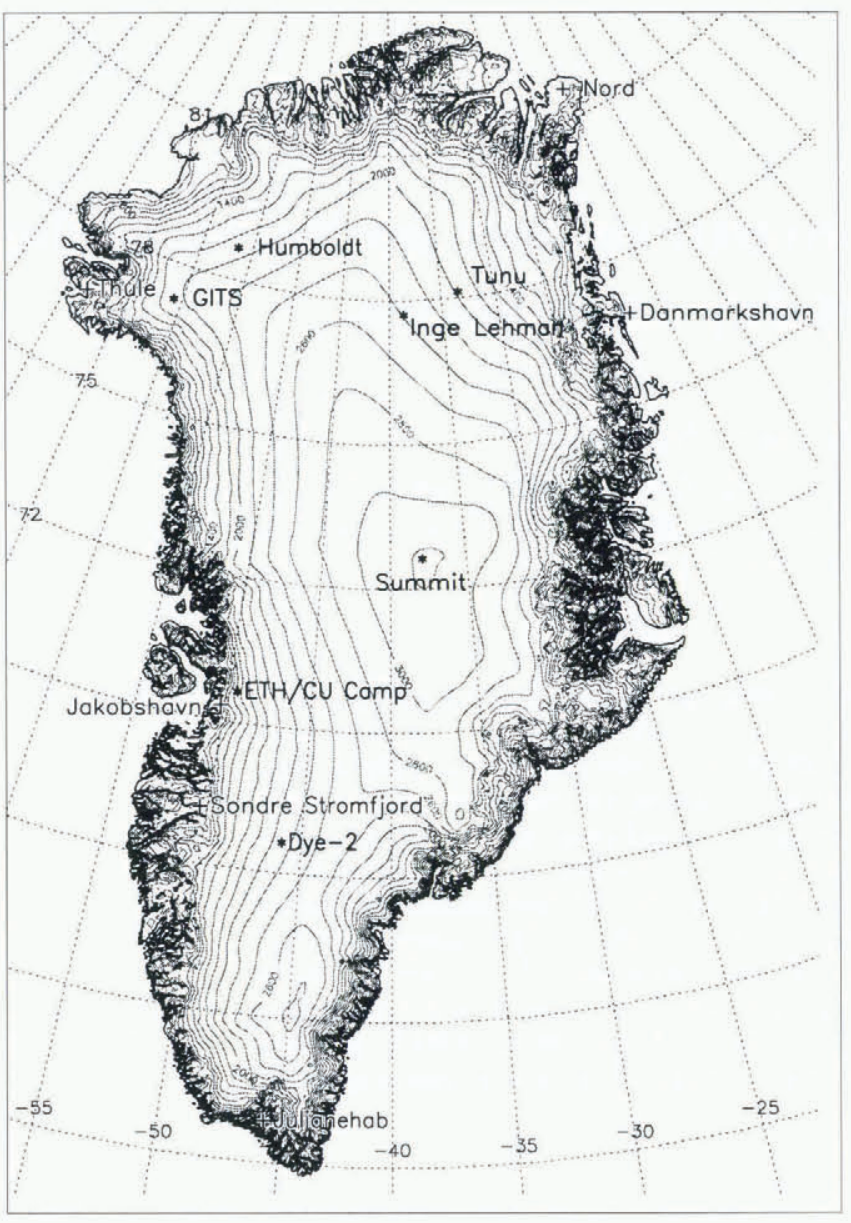

Fig. 1. The Greenland ice sheet. Snow-grain profiles were taken from Inge-Lehman (Gow, 1971). Tunu was the site of the 1996 PARCA field campaign from which snow-structure characteristics were also determined and an automatic weather station (AWS) was set up. Climatological and accumulation data from this AWS will be used for future model refinement. Summit is the site from which ice-core data were provided (Bolzan and Strobel, 1994).

the purposes of assessing the relative impacts of hoar development and accumulation, examining the emissivities is sufficient. Incorporation of firn-temperature and atmospheric conditions will be the subject of future modeling efforts as those data become available.

\section{RADIATIVE TRANSFER IN THE FIRN}

Emission in the snowpack is governed by the integro-differential radiative-transfer equation, given as (Comiso and others, 1982):

$$
\begin{aligned}
I(\theta) & =I(\tau, \theta) e^{\frac{-\tau}{\mu}}+\int_{\tau}^{0}\left\{\varepsilon\left(\tau^{\prime}\right) B\left[T\left(\tau^{\prime}\right)\right] \frac{e^{\frac{-\tau}{\mu}}}{\mu}\right\} \mathrm{d} \tau^{\prime} \\
& +\frac{1}{2}\left\{\int_{\tau}^{0}\left\{\int_{0}^{\pi} P\left(\theta^{\prime}\right) I\left(\tau^{\prime}, \theta^{\prime}\right) \sin \theta^{\prime} \mathrm{d} \theta^{\prime}\right\} \omega_{0}\left(\tau^{\prime}\right) \frac{e^{\frac{-\tau}{\mu}}}{\mu}\right\} \mathrm{d} \tau^{\prime}
\end{aligned}
$$

where $I(\theta)$ is the intensity (energy flux per unit wavelength per unit solid angle), $\theta$ refers to the zenith angle, $\tau$ is the optical depth, $\varepsilon$ is the volumetric emissivity, $B$ is the RayleighJeans approximation of the Plank function, $T$ is temperature; $\omega_{0}$ is the single-scattering albedo, $\tau^{\prime}$ and $\theta^{\prime}$ are the integration variables for depth and scattering angle, respectively, $\mu=\cos (\theta)$ and $P(\theta)$ is the scattering-phase

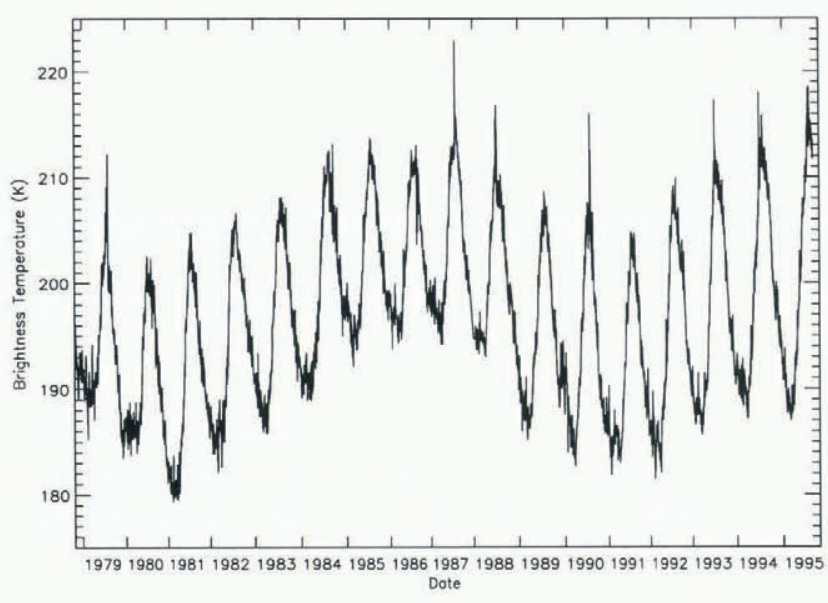

Fig 2. Microwave brightness temperatures for the 18 and $19 \mathrm{GHz}$ vertical polarization of SMMR and SSM/I, respectively, for fanuary 1979 through August 1995 at the Tunu site. A significant increasing trend is evident between the years 1981 and 1985, which has been hypothesized to be related to accumulation changes (Steffen and others, 1993).

function, which describes the angular distribution of the scattered energy.

Each of the terms on the right side of the radiative-transfer equation represents a specific contribution to the upwelling intensity of the microwave energy from the surface of the snowpack. The first is simply the transmission of upwelling intensity from a given optical depth, $\tau$, to the surface. This term accounts for the extinction of upwelling intensity below the depth of a medium as it passes through the medium to the surface (by multiplication by the extinction term, $\left.e^{-\tau / \mu}\right)$. The second is the source term, which represents the contribution of thermally emitted radiation from within the medium. Included in this term are the emissivity, to account for the gray body nature of the medium and the extinction factor, to account for losses within the medium. Finally, the third term represents the directionally variable (anisotropic) scattering of radiation within the medium. This term includes the phase function, single-scattering albedo and exponential extinction factor. Assuming independent scattering, the upwelling intensity is simply the sum of all of these components.

Once intensity is calculated, the emissivity can be determined according to

$$
\varepsilon=\frac{\lambda^{4} I}{2 c k T_{\mathrm{p}}}
$$

where $\lambda$ is the wavelength, $c, k$ and $T_{\mathrm{p}}$ are the speed of light, Boltzmann's constant and the physical temperature of the snowpack, respectively. According to the Rayleigh-Jeans approximation (Equation (1)), at microwave frequencies, the term $\lambda^{4} I / 2 c k$ is the brightness temperature.

\section{RADIATIVE-TRANSFER MODEL DEVELOPMENT}

The details of the model are discussed in the items below, but in summary, we developed a 20 layer model (Fig. 2) of the top $25 \mathrm{~m}$ of firn at a temperature of $233 \mathrm{~K}$ (approximate winter temperature at the Tunu site). The top three layers represent 1 year's worth of snowfall, with the middle of the three being the hoar layer and the bottom and top being the snowfall before and after the hoar formation, respectively. These upper layers were established to isolate the hoar and allow for variation in accumulation and hoar-layer thick- 
ness. The grain-sizes of each of these three layers were made to vary inversely with accumulation, as discussed in items 5 and 14 below. The next 17 layers represent the remainder of the firn down to $25 \mathrm{~m}$ and all layers are assumed horizontally homogeneous. Next, the single-scattering albedo and optical thicknesses are calculated (after Zwally, 1977) for each layer. These values are then input into a Discrete Ordinate Radiative Transfer (DISORT) model (Stamnes and others, 1988) adapted for $19.35 \mathrm{GHz}$ vertically polarized emission at an angle of $53^{\circ}$. The bulk emissivity is then calculated according to Equation (3) (for $T_{\mathrm{p}}=233 \mathrm{~K}$ ). The model is run for various accumulation rates and hoar-layer thicknesses, and the emissivity characteristics are assessed.

To insure consistency with previous work, all assumptions and characteristics of the model are either taken directly from or derived from Chang and others (1976), Zwally (1977), Comiso and others (1982) or Van der Veen and Jezek (1993). They are summarized in Table 1 and are discussed in more detail below.

\section{Model assumptions and parameters}

\section{(1) Horizontal homogeneity}

The snowpack is assumed to be horizontally homogeneous and structured, as shown in Figure 3. While there is in fact significant vertical variability in the structure of the firn, with the pinching-and-swelling of the layers, these layers are qualitatively maintained even over large distances (Steffen and others, 1995). Since the model results are intended to aid in the interpretation of the SMMR and SSM/I data products (both the standard polar-stereographic and the EASE-

\section{Table 1. Model assumptions and characteristics for nominal conditions on the ice sheet (mean annual accumulation of $100 \mathrm{~mm}$ w.e. (Ohmura and Reeh, 1991) and $1.5 \mathrm{~cm}$ thick hoar layer). Also given are the sources of the assumptions made and the sensitivity of the modeled microwave emissivity to each assumption or characteristic}

\begin{tabular}{|c|c|c|}
\hline Assumption/characteristic & Sources ${ }^{*}$ & Sensitivity $^{*}$ \\
\hline Horizontal homogeneity & $1,2,3,4$ & Variable \\
\hline Absorption coefficient & 2 & Moderate \\
\hline Isothermal snowpack & 2,3 & None \\
\hline Grain-size distribution & 2,3 & Moderate \\
\hline Constant annual growth rate & 2 & Low \\
\hline $19.35 \mathrm{GHz}$ frequency & $1,2,3,4$ & Model constraint \\
\hline Rayleigh scattering & $1,2,3,4$ & $\begin{array}{l}\text { None except for hoar } \\
\text { (discussed in text) }\end{array}$ \\
\hline Independent scattering & $1,2,3,4$ & $\begin{array}{l}\text { Empirically accounted } \\
\text { for (see below) }\end{array}$ \\
\hline Scattering coefficient & $1,2,3,4$ & Low \\
\hline Optical depth & $2,3,4$ & Low \\
\hline Single-scattering albedo & $1,2,3,4$ & Extreme \\
\hline Winter conditions & This study & Model constraint \\
\hline Hoar at center of annual layer & This study & Low \\
\hline Hoar radius & This study & Low \\
\hline Hoar-scattering characteristics & 2,This study & Low \\
\hline Emission angle & This study & Model constraint \\
\hline Vertical polarization & This study & Model constraint \\
\hline
\end{tabular}

\footnotetext{
Source references: 1. Chang and others, 1976; 2. Zwally, 1977; 3. Comiso and others, 1982; 4. Van der Veen and Jezek, 1993.

+ Sensitivity terms are calculated based on the impact of a $20 \%$ change in the assumed value (where appropriate). The convention is as follows: 0 $2 \%$ change in calculated emission - low; 2 - 5\% change - moderate; 5 $10 \%$ - high; $10-20 \%$ - extreme.
}

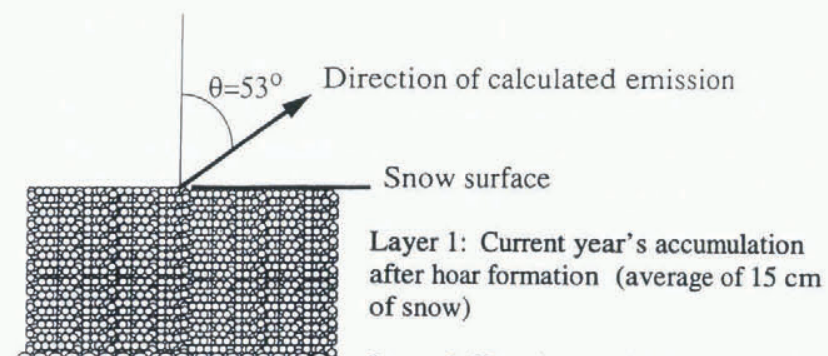

Layer 2: Hoar (mean thickness $=1.5 \mathrm{~cm})$

Layer 3: Current year's accumulation before hoar formation (average of 15 $\mathrm{cm}$ of snow)

Layers 4 - 20: extending to $25 \mathrm{~m}$ depth.

The cube of the radius increases linearly with depth.

Fig 3. Snow struclure used in the radiative-transfer model. The structure represents winter conditions at Inge-Lehman, in which the summertime hoar is sandwiched between the spring and fall snow. The grain growth os depth relationship was based on observations of Gow (1971).

Grid products), which are mapped on to $25 \mathrm{~km} \times 25 \mathrm{~km}$ gridcells, the effects of vertical variability are assumed to be mitigated by averaging over these large distances. By assuming horizontal homogeneity, the intensity is considered to be azimuthally independent.

\section{(2) Absorption coefficient}

The absorption coefficient $\left(\gamma_{\mathrm{a}}\right)$ is set equal to $0.038 \mathrm{~m}^{-1}$. Zwally (1977) used a value of $0.15 \mathrm{~m}^{1}$ but Comiso and others (1982) showed that, for the polar firn, $0.038 \mathrm{~m}^{-1}$ is more consistent with the theoretically derived values from the Debye equation (Hobbs, 1974), and it also agrees with estimates made from a compilation by Evans (1965) of measured loss tangents Comiso and others, 1982). Agreement between the different estimates is best at approximately $233 \mathrm{~K}$, which is a reasonable winter-firn temperature at Tunu. Furthermore, $\gamma_{\mathrm{a}}=0.038 \mathrm{~m}^{-1}$ shows an excellent fit with empirical data for various sites in Greenland and Antarctica, particularly for the Inge-Lehman station in northeast Greenland Comiso and others, 1982); therefore, for the range of temperatures in the emitting region, this value is used.

\section{(3) Isothermal snowpack}

In reality, the snowpack is not isothermal, even as an annual average (Van der Veen and Jezek, 1993). However, at microwave wavelengths, the intensity (in Equation (3)) is a linear function of $T_{\mathrm{p}}$; thus, if an isothermal snowpack is assumed, the resulting emissivity is not temperature-dependent, except to the extent that single-scattering albedo and absorption coefficient depend on temperature. Since we are limiting our analysis to the study of the impact of changes in accumulation and hoar formation on the emissivity rather 
than $T_{\mathrm{b}}$, only an approximate average temperature is needed rather than the detailed vertical temperature structure. If brightness temperatures were to be determined, or accumulation estimates were to be made by inverting the model, the temperature profile would be necessary. In our analysis, we assume an average firn temperature of $233 \mathrm{~K}$, which is appropriate for the region in winter.

\section{(4) Grain-size distribution}

The cube of grain radius $(r)$ can be modeled as a linear function of depth $(z)$ :

$$
r^{3}=r_{0}^{3}+S z
$$

where $r_{0}^{3}$ is equal to 0.0278 and $S$ is equal to 0.0202 for $z$ in meters, and $r_{0}^{3}$ in $\mathrm{mm}^{3}$ (Zwally, 1977). This relationship is primarily dependent on mean annual temperature and accumulation rates (Gow, 1969).

\section{(5) Constant annual grain-growth rate}

The size of the particle at the bottom and top of the current year's snowfall is assumed not to vary from year to year; thus, with varying accumulation, the grain-size vs depth relationship for the current year's snowfall ( $S$ in Equation (4)) varies inversely with the accumulation rate (Zwally, 1977). For layers 1 and 3 (in Fig. 3), $r$ is related to $z$ according to:

$$
r^{3}=r_{0}^{3}+S\left(\frac{\bar{A}}{\bar{A}}\right) z
$$

where $\bar{A}$ is the mean annual accumulation rate (in snow height) and $A$ is the depth of accumulated snow for the current year. Using this scaling factor, the effects of increased or decreased snowfall on the grain-size profile in the current year's snow layer are accounted for. The scaling factor becomes infinite as $A$ approaches zero; however, for accumulation rates greater than or equal to $10 \mathrm{~cm}$ in height of snow a ${ }^{1}$ (one-third of $\bar{A}$ for the region), the values are reasonable. This relationship insures that volume grain growth is linear with time rather than with depth. A similar scaling factor is applied to the hoar and is discussed in item 14 below.

\section{(6) $19.35 \mathrm{GHz}$ frequency ( $1.55 \mathrm{~cm}$ wavelength)}

The radiative characteristics of polar firn at this frequency are of great interest for interpretation of SSM/I microwave brightness temperatures. The closest channel on the SMMR instrument is $18 \mathrm{GHz}$ but, through the application of regression coefficients (Jezek and others, 1991), they can be modified to represent their $19.35 \mathrm{GHz}$ equivalent. While there is certainly additional important information in the $37 \mathrm{GHz}$ frequency (which are also channels on SMMR and SSM/ I), the $19.35 \mathrm{GHz}$ was chosen because it allows for the assumption of Rayleigh and independent scattering. The physics of the model would be significantly more complex for the higher frequencies, and many of the assumptions made in previous work by Zwally (1977), Comiso and others (1982) and Van der Veen and Jezek (1993) would not apply.

\section{(7) Rayleigh scattering}

For $19.35 \mathrm{GHz}$, particles on the order of $1.5 \mathrm{~mm}$ or less in diameter satisfy the Rayleigh scattering criterion. Thus, the assumption is applicable to the snowpack. The Rayleigh criteria are not quite satisfied by larger hoar particles; however, Rayleigh scattering is still assumed, with the acknowledgment that the amount of scattering is slightly underestimated. The implications of this assumption are discussed at the end of this section.

\section{(8) Independent scattering}

The snow grains are assumed to scatter independently Chang and others, 1976; Zwally, 1977; Comiso and others, 1982; Van der Veen and Jezek, 1993). Because of the dense packing of the snow, however, there are some coherent scattering effects and the assumption results in an overestimate of the scattering (Zwally, 1977; Comiso and others, 1982). This overestimate is one factor which is accounted for by the empirical correction term, $f$, discussed in item 9 below.

\section{(9) Scattering coefficient}

The scattering coefficient $\left(\gamma_{\mathrm{s}}\right)$ varies with radius $(r)$ according to the formula:

$$
\gamma_{\mathrm{s}}=f(1.8 r)^{3}
$$

where the empirically derived correction factor, $f$, is assumed to be equal to 0.3 (Comiso and others, 1982). It accounts for the overestimating of scattering due to various assumptions in the derivation of the scattering coefficient, primarily that of independently scattering, spherical particles. Alley (1987) demonstrated that snow grains in polar firn can be approximated as prolate spheroids whose major axis is oriented vertically. However, the axial ratio is largely variable, ranging from 1 (indicating spherical particles) to 1.4 (elongated particles) (Alley, 1987). Because of the variability, and the fact that previous models have shown that the $f$ term compensates for shortcomings of this and other assumptions, we also assume scattering characteristics of spherical particles with an empirically derived adjustment factor. A comparison with seven stations in Greenland and Antarctica showed that, for $f=0.3$, the scattering term yields an excellent fit $(R=0.986)$ to empirical data (Comiso and others, 1982). Therefore, in this study $f$ is assumed to be 0.3 .

Though not explicitly input into the model, the real and imaginary parts of the index of refraction are implicit in the calculation of the scattering and absorption coefficients. The coefficients described in the above assumptions correspond to an index of refraction $(n)$ whose real component $\left(n^{\prime}\right)$ is 1.78 , and imaginary component $\left(n^{\prime \prime}\right)$ is $0.00014 i$.

\section{(10) Optical depth $(\tau)$}

The optical depth of any given layer, the top and bottom of which are at depths of $z_{1}$ and $z_{2}$, respectively, is (from Zwally, 1977, Equation (23)):

$$
\tau(z)=\int_{z_{2}}^{z_{1}}\left[\gamma_{\mathrm{a}}+\gamma_{\mathrm{s}}(z)\right] \mathrm{d} z .
$$

Under normal accumulation conditions, $\bar{A} \approx 100 \mathrm{~mm}$ w.e. $\mathrm{a}^{-1}$ (Ohmura and Reeh, 1991), the typical optical depth for 1 year's accumulation is approximately 0.027 . The optical depths of hoar layers range from 0.006 to 0.184 for $1 \mathrm{~mm}$ and $3 \mathrm{~cm}$ layer thicknesses, respectively; thus, according to the model, a hoar layer of less than $0.5 \mathrm{~cm}$ can be of similar optical thickness to lyear's worth of accumulation, in lowaccumulation areas such as Tunu. In higher-accumulation areas, the optical thicknesses of the annual snowfall would be significantly greater in relation to the hoar.

\section{(11) Single-scaltering albedo $(\omega)$}

The single-scattering albedo is given by

$$
\omega_{0}=\frac{\gamma_{\mathrm{s}}}{\gamma_{\mathrm{a}}+\gamma_{\mathrm{s}}} .
$$

Table 1 indicates that the results are highly sensitive to single-scattering albedo; a $20 \%$ change in $\omega_{0}$ alters the 
emissivity by $19 \%$. Fortunately, the differences between the magnitudes of $\gamma_{\mathrm{a}}\left(0.038 \mathrm{~m}^{-1}\right)$ and $\gamma_{\mathrm{s}}$ (on the order of $0.2 \mathrm{~m}^{-1}$ for most of the emitting area of the snowpack) are such that changes in $\gamma_{\mathrm{a}}$ are not of major impact on $\omega_{0}$, and changes in $\gamma_{\mathrm{s}}$ to some extent cancel out in the ratio.

\section{(12) Winter conditions}

The model stratification is as shown in Figure 3, which is representative of winter conditions in the absence of wintertime hoar formation (discussed by Steffen and others, in press). Winter conditions are used because any effects attributable to insolation do not influence observed wintertime brightness temperatures.

\section{(13) Hoar at the center of the annual layer}

The situation of the hoar at the center of the annual layer arises from two assumptions. The first is that the amount of snow before the formation of hoar in a given year is equal to the amount of snow that falls after the formation of hoar. This assumption was based on an analysis of shallow icecore data provided by J. Bolzan of the Byrd Polar Research Center at The Ohio State University. A 24 year comparison of winter-to-summer accumulation and summer-to-winter accumulation showed no bias one way or the other between winter/spring and summer/fall snowfall over the period studied. There is most likely some seasonal variation from year to year but the ice-core data suggest that over long time periods they average out. The half-year estimates were determined from winter and summer peaks in the stable oxygen-isotope ratios for the years 1964-87 at nine sites on a $150 \mathrm{~km} \times 150 \mathrm{~km}$ grid around Summit, Greenland (described in Bolzan and Strobel (1994)). The conditions at Tunu and Inge-Lehman may be a little different than Summit but the Bolzan and Strobel data are believed to reflect the general pattern. In addition, modeled precipitation values of Bromwich and others (1993) show that the seasonal cycles of precipitation are centered roughly around early July (in the maximum) and also late January (in the minimum). Thus, it is reasonable to assume in this analysis that summertime hoar layers will be at or near the center of the annual layer.

The second assumption is that the hoar forms at or near the surface during the summer, as is the case at high elevations due to intense insolation (Alley and others, 1990). Hoar also forms at the interface between a crust layer and vertically adjacent low-density snow in the presence of a strong thermal gradient. However, for the purposes of isolating the effects of a hoar layer on the microwave emission, only a single layer was included at the center of the annual layer, with the concession that hoar development closer to the surface will affect more strongly the emission and one further from the surface will have less of an impact. The results are not significantly influenced by where in the snow layer the hoar lies; a 20\% error in this estimate results in less than $1 \%$ change in the snow emission. For a $233 \mathrm{~K}$ firn temperature and an emissivity of 0.7 , this corresponds to a $1.6 \mathrm{~K}$ change in brightness temperature.

\section{(14) Hoar radius ( $\left.r_{\text {hoar }}\right)$}

The mean hoar radius in the model is $1.5 \mathrm{~mm}$. This is typical of observed values at the Tunu site. As with near-surface grain-sizes, the size of the hoar particles is assumed to vary inversely with the accumulation rate according to:

$$
r_{\text {hoar }}^{3}=\left(\frac{\bar{A}}{\bar{A}}\right)\left(\bar{r}_{\text {hoar }}\right)^{3}
$$

in order to account for the hoar-size variability with changing accumulation.

\section{(15) Hoar-scaltering characteristics}

The dependence of scattering on the factor $(1.8 r)^{3}$ in Equation (6) is valid for grain-sizes of less than $1 \mathrm{~mm}$ radius but, for the larger hoar particles, $\gamma_{\mathrm{s}}$ is taken to be $(1.82 r)^{3}$ (Zwally, 1977). The hoar is assumed to be in the Rayleigh regime (the implications are discussed at the end of this section) and only a single layer of hoar is included near the surface of the model. Hoar layers may be present for many years before metamorphism and causes them to become nearly indistinguishable from the surrounding snow but this investigation is intended to assess the impact of the most recently formed hoar (from the preceding summer). Subsequently, only one layer was modeled. Furthermore, the contribution of deeper hoar layers from previous years is less than that of the near-surface layer by virtue of their depth.

\section{(16) Emission angle}

Intensity is calculated for an angle of $53^{\circ}$ from the zenith, which is the scan angle of the SSM/I instruments. For estimates of accumulation from brightness temperatures, the emission characteristics at the instrument-observation angle must be evaluated. The SMMR scan angle is closer to $50^{\circ}$ but as with wavelength the brightness temperatures can be adjusted to reflect their $53^{\circ}$ equivalent with the appropriate regression coefficients (Jezek and others, 1991).

\section{(17) Vertical polarization}

The $50^{\circ}$ and $53^{\circ}$ viewing angles are near the Brewster angle for snow (Hollinger and others, 1987). As a result, the effects of the layered nature of the firn on vertically polarized emission are at their minimum, thus maximizing the emissivity dependence on grain-size distribution. This grain-size distribution is presumed to be closely related to accumulation and hoar development.

The vertical component of the Rayleigh-scattering phase function for spherical particles is (from Goody, 1964):

$$
P(\theta)=\frac{3}{4}\left[(\cos \theta)^{2}\right] \text {. }
$$

This phase function is incorporated into the discrete ordinate model as the coefficients of a Legendre polynomial expansion.

\section{Assumption validity for hoar layer}

The validity of most of these assumptions has been discussed by Zwally (1977) and Comiso and others (1982) for the polar firn. Those that have not, have been discussed above. An issue that needs to be addressed in this analysis is the extent of applicability of these assumptions to a model in which a hoar layer is present. One error source may be the far-field assumption, or the treatment of the particles as independent scatterers. The applicability of the far-field approximation is dependent on the packing density of the medium or the iceair ratio. The lower this ratio, the more valid the approximation. In the hoar layer, the large particles actually result in reduced packing density as compared to the rest of the snow pack. Thus the far-field assumption is in fact more valid 
for the hoar layer than it is for the rest of the snowpack. Since $f$ accounts for some deviation from the far-field conditions, the hoar effects may be slightly underestimated and are likely to be greater than predicted by the model.

Another important consideration is the assumption of Rayleigh scattering in the hoar layer. The scattering is actually somewhere between the Mie regime, where particle size is on the order of one wavelength, and the Rayleigh regime, where the particle is less than one-tenth of the wavelength. Our field observations show the hoar particles to be on the order of $3 \mathrm{~mm}$ in diameter, which is approximately one-fifth of the modeled wavelength; thus, the scattering is more closely related to Rayleigh than to Mie. The associated errors are likely to be small but their effects on the model should be considered.

Mie scattering is more in the forward direction than is Rayleigh, which is more symmetric about the horizontal plane, $\theta=90^{\circ}$ (Henderson-Sellers and Robinson, 1991). This effect would suggest that the Rayleigh assumption overestimates scattering. However, calculation of the Henyey-Greenstein asymmetry parameter $(g)$, gives a result of $g=0.082$. A positive value of $g$ indicates preferentially forward scattering (for $g=1$, scattering is purely forward) and a negative value of $g$ indicates preferentially backward scattering (for $g=-1$, scattering is purely backward). In the case of symmetric scattering, $g$ is equal to zero. The fact that the magnitude of the calculated value of $g$ is so low means that scattering is only very slightly biased in the forward direction and it is of little consequence in the overall results.

\section{RESULTS AND DISCUSSION}

In order to validate the model, it was run for the same input conditions as those of Comiso and others (1982) and yielded the same results to within a few tenths of a percent. This is within the limits of the two different solution methods (doubling-adding vs discrete ordinates). An additional test case was run for a pure scattering medium with a Rayleighphase function (Chandrasekhar, 1960; Comiso and others, 1982) and again the results were within the limits of the different solution methods. The model was then run for various hoar-layer thicknesses and accumulation rates. The results of these runs are presented below and the importance of hoar, in addition to accumulation, in the microwave emission is presented and discussed.

It is important to bear in mind that the observed $18 \mathrm{~V} /$ $19 \mathrm{~V}$ wintertime brightness temperatures for the Tunu region are on the order of $195 \mathrm{~K}$ (Fig. 2). If we assume an atmospheric contribution of $25 \mathrm{~K}$, the surface $T_{\mathrm{b}} \mathrm{s}$ are in the vicinity of $170 \mathrm{~K}$. Thus, emissivity need only change by as little as $1 \%$ to cause a detectable change in brightness temperature $\left(1 \%\right.$ change in $\varepsilon$ results in a $1.7 \mathrm{~K}$ change in $T_{\mathrm{b}}$ ).

\section{Emissivity and accumulation}

The dependence of emissivity on accumulation and hoar development is shown in Figure 4. The results indicate that an increase in accumulation causes an increase in microwave emissivity (as was shown by Zwally (1977)). This is to be expected since greater accumulation rates result in increased signal contribution from the highly emissive smaller grains of relatively new snow. In addition, the sensitivity of emissivity to accumulation rate is greatest in the areas of
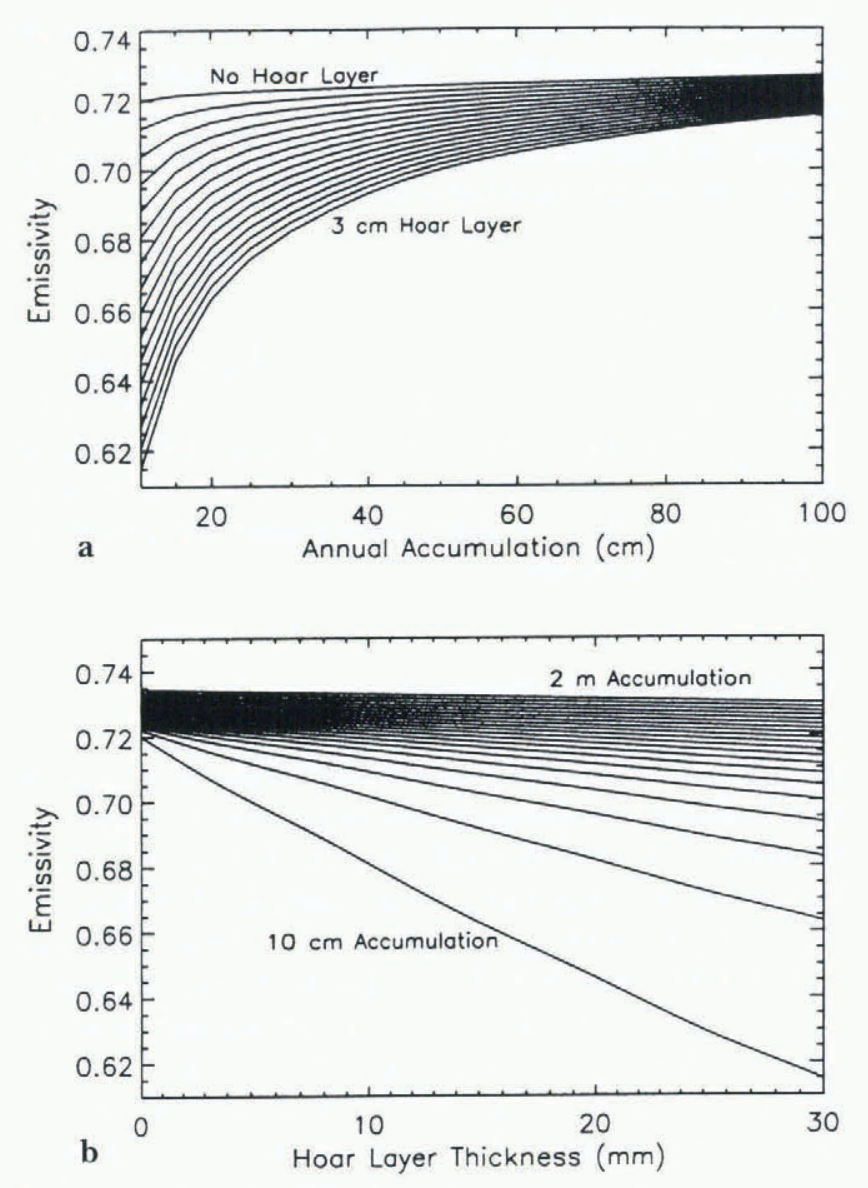

Fig 4. Dependence of microwave emissivity on accumulation and hoar thickness at Tunu and Inge-Lehman. Accumulation rates are given in meters (or centimeters) of snow, and hoarlayer thickness is also given in centimeters of hoar. Appropriate density values for conversion to water equivalents is $380 \mathrm{~kg} \mathrm{~m}^{-3}$ for the snow, and $300 \mathrm{~kg} \mathrm{~m}^{-3}$ for the hoar. The isolines in ( a) are at intervals of $2 \mathrm{~mm}$, while those in (b) are at $10 \mathrm{~cm}$ intervals. The sensitivity is greatest when accumulation is low and for thick hoar layers.

the lowest accumulation. This occurs because, in high accumulation areas, snowfall from the current year already comprises a large part of the signal and masks out much of the contribution from the previous year's snow; thus, changes in snowfall do not significantly impact the signal. Consider, for example, the extreme case of an area with $5 \mathrm{~m}$ of snowfall per year. Each year, the $19 \mathrm{GHz}$ microwave emission will be nearly totally emitted from the current year's snowfall. Thus, a doubling of snowfall will not result in much of a change in the signal.

Conversely, in areas with little accumulation, a doubling of snowfall will result in much more of the signal coming from the current year's more highly emitting snow than in previous years. As a result, the sensitivity to accumulation variations is greater in these low-accumulation regions. Typical rates of accumulation for the vicinity of Tunu and Inge-Lehman are approximately $30 \mathrm{~cm}$ of snow per year (based on Ohmura and Reeh (1991), and assuming a density of the top snow layer of $380 \mathrm{~kg} \mathrm{~m}^{3}$ ). Also, it is very likely that in such low-accumulation areas, snowfall may vary from 50 to $200 \%$ of the annual mean (Mosley-Thomson and others, 1995).

The accumulation-emissivity relationships are consistent with what has already been shown qualitatively by Zwally (1977). They also explain in part why the observed 
brightness temperature trends (Steffen and others, 1993) are strongest in the low-accumulation areas. However, what is different and of great importance to the future of microwave modeling and for the interpretation of long-term passive microwave satellite data of the Greenland ice sheet is the dependence and sensitivity of this relationship to the presence of hoar.

\section{Emissivity and hoar-layer thickness}

The microwave emissivity is very sensitive to the hoarlayer thickness itself. According to the model, the presence of a $1.5 \mathrm{~cm}$ thick hoar layer, which is typical of the region, based on our observations at Tunu, reduces the emissivity by $2.8 \%$. Assuming the mean wintertime $19 \mathrm{GHz}$ vertically polarized brightness temperature is $170 \mathrm{~K}$, a $1.5 \mathrm{~cm}$ hoar layer is responsible for a $5 \mathrm{~K}$ reduction. In other words, if the hoar were not present, the brightness temperature would be $175 \mathrm{~K}$. Considering observed hoar layers at Tunu ranged from $1 \mathrm{~mm}$ to $4 \mathrm{~cm}$ in thickness, impact of hoar on brightness temperature can be major.

Furthermore, according to the model, variations in hoar thickness of a millimeter have roughly the same impact on the emissivity as a few centimeters of accumulation changes. In terms of percentages, a $10 \%$ increase in hoar-layer thickness may impact the signal by as much as a $15 \%$ change in accumulation. The large optical depth and the high scattering characteristics result in a significant hoar contribution to the microwave emission.

Finally, the sensitivity of microwave emissivity to hoar development is lowest in the regions of maximum snowfall. This can be explained by the fact that high-accumulation rates more effectively mask the hoar signal and thus reduce the sensitivity of the overall emission to the hoar development. Such an effect further explains the inverse relationship between $T_{\mathrm{b}}$ "trend strength" and mean accumulation rates (Steffen and others, 1993).

\section{Accumulation and hoar: combined effects}

While the emissivity does show some variation with accumulation, the magnitude of the relationship is weak without the consideration of hoar (as indicated by the shallow slope of the top curve in Figure $4 a$ ). In the absence of hoar, even a $3 \mathrm{~K}$ rise in $T_{\mathrm{b}}$ in the Tunu region, which was observed for six consecutive years in the SMMR data (Steffen and others, 1993 cannot be caused by accumulation variations alone. When considered with a $1.5 \mathrm{~cm}$ hoar layer in the snowpack, however, less than a doubling $(60 \mathrm{~cm}$ annual accumulation, or approximately $230 \mathrm{~mm}$ water equivalent) of accumulation will cause such a rise in $T_{\mathrm{b}}$. The relationship indicates that, without the hoar, accumulation changes would be undetectable with the $19 \mathrm{~V}$ channel. In addition, the thicker the hoar layer, the stronger the relationship between accumulation and microwave emissivity.

This strengthened relationship and its dependence on hoar-layer thickness is most likely attributable to the high scattering characteristics of the hoar layer, which cause a significant extinction of the upwelling radiation from the snow below. It has been well documented that depth hoar in snowpacks on land surfaces are effective volume scatterers and significantly diminish upwelling radiation from below (Mätzler and others, 1980; Hall and others, 1986, 1991; Sturm and others, 1993). Our model results demonstrate that the same is true for firn. As a result, in the pre- sence of a thick hoar layer, more of the signal comes from the near-surface layers of snow than the deeper parts of the snowpack; thus, the sensitivity of the signal to changes in snow near the surface is greater.

Additional information about the accumulation variability is contained in the $37 \mathrm{GHz}$ data, which would be more sensitive because of its smaller wavelength and lower penetration/emission depth. However, the sensitivity of emission at $37 \mathrm{GHz}$ to hoar development would also be higher, and probably to a greater relative degree, since the sizes of hoar particles are considerably closer to the wavelength. Thus, estimating the accumulation from the $37 \mathrm{GHz}$ emission requires an even greater assessment/parameterization of the hoar development.

Significant progress has been made in identifying hoar formation events on the Greenland ice sheet using the ratio of $37 \mathrm{GHz}$ vertical and horizontal brightness temperatures (Shuman and others, 1993). However, a method of quantitatively estimating the amount of hoar has not yet been developed. This would require occupation of the modeled site during a hoar-formation event and continuous observations of the snow accumulation, sub-surface temperatures and atmospheric conditions. Automatic Weather Stations (AWSs) that measure these parameters are already in place at a number of sites on the ice sheet, and occupation of one or more of these sites during a hoar-formation event, in conjunction with continued monitoring with the AWS, would allow for comparisons between microwave emission and snow accumulation over time. This is expected to be the subject of future field work.

Pending such field work, a possible proxy may be to monitor the $37 \mathrm{~V} / 37 \mathrm{H}$ brightness temperature ratio throughout the summers for the modeled location. Sustained decreases, which are indicative of hoar-formation events (Shuman and others, 1993), could be identified each year and used in a relative sense to characterize the degree of hoar development. This is a simplistic approach to a complex problem but could represent a first step toward appropriately incorporating hoar layers in the firn models. Because the intent of the research in this paper is to assess the relative importance of hoar in the firn, the development of such a parameterization is beyond the scope of this work. It would, however, be a necessary step towards relating accumulation/hoar formation to changes in brightness temperature.

\section{The observed trends: other explanations}

The model results indicate that, in the absence of hoar, accumulation alone could not account for the observed $18 \mathrm{~K}$ trend in $T_{\mathrm{b}}$ observed in the 1980s (Fig. 2). We have demonstrated that significant changes in $T_{\mathrm{b}}$ can be caused by variations in hoar-layer thickness alone or in conjunction with changes in accumulation rates. However, considering the magnitudes of the effects of hoar and accumulation, it is likely that there are additional phenomena contributing to the observed changes. These possibilities, though not analyzed in this paper, should be mentioned. One is atmospheric contributions. Energy emitted by the atmosphere contributes to the $T_{\mathrm{b}}$ observed by the satellite. If cloudiness were to increase steadily, between 1979 and 1987, or if more warm air were advected to the interior of the ice sheet, it is possible that $T_{\mathrm{b}}$ could also increase. However, comparison to coastal climate stations shows no atmospheric warming. 
In addition, the trends were observed to be as strong in the winter as in the summer. Because the region examined is at approximately $2000 \mathrm{~m}$ elevation, winter cloud cover is assumed to be very low and its variability is not likely to account for such a large brightness temperature increase.

Another possibility is that, despite being well outside the accepted melt regime (Benson, 1962; Mote and Anderson, 1995; Abdalati and Steffen, 1997), the trend may have to some extent been caused by a brief anomalous melt event. Close examination of Figure 2 shows several spikes in the $18 \mathrm{~V} / 19 \mathrm{~V}$ brightness temperatures in some years, the most significant of which is the summer of 1979. Though they are short in duration, these spikes are indicative of some degree of melting (Mote and others, 1993; Zwally and Fiegles, 1994). Such an event would form a thin melt crust which, once refrozen, would serve to reduce overall emissivity. The pores between grains would disappear and the scattering of upwelling electromagnetic energy would increase significantly. The result would be a lower brightness temperature after refreezing of the melt crust. As subsequent melt-free years passed, and the melt layer were buried more deeply, its effects would be expected to diminish.

The most significant of these melt spikes occurred in 1979 and, as expected, the brightness temperatures in the following winter dropped approximately $4 \mathrm{~K}$. However, there are no signs of a melt event in the summer of 1980 , yet the mean $T_{\mathrm{b}}$ of the following year dropped approximately $5 \mathrm{~K}$. Furthermore, other years which show similar melt spikes do not show a strong correlation with decreased $T_{\mathrm{b}} \mathrm{s}$ in the subsequent winters. Thus, there is a level of variability which is not attributable to melt events. We therefore assume that the variation is a result of some combination of hoar formation (or other metamorphic factors causing increased grain-size), accumulation, atmospheric and temperature effects, and surface melting.

\section{GONGLUSION}

With a radiative-transfer model, we were able to examine the emission behavior of snow under changing conditions of accumulation and hoar development. The direct dependence of microwave emission on accumulation rates in the dry-snow area of the Greenland ice sheet is of some significance; however, the emissivity depends as much, if not more, on the variable thicknesses of the hoar layers. As a result, accumulation estimates based on passive microwave observations require successful parameterization of the hoar-formation characteristics. Until this hoar development can be adequately parameterized, the detection of interannual variations in accumulation using $19 \mathrm{GHz}$ brightness temperatures will not be possible.

Furthermore, the model demonstrates that the microwave emission from the firn is much more sensitive to changes in accumulation and hoar development in the areas of little precipitation, such as the northeast region of the ice sheet. This explains why the observed brightness temperature trends in the SMMR time series of the dry-snow regions of the ice sheet were strongest near Tunu and weaker farther away (Steffen and others, 1993). For these reasons, the Tunu area is most useful for examining the manifestation of surface characteristics in the microwave emission from the Greenland ice sheet.

\section{ACKNOWLEDGEMENTS}

This research was supported by NASA Polar Program grant NAWP-2158, NASA grant NAS5-32484 and NSF grant OPP9423530. The passive microwave satellite data were provided by the National Snow and Ice Data Center (NSIDC) in Boulder, Colorado. We should also like to acknowledge the contributions of J. Bolzan, Byrd Polar Research Center at The Ohio State University, who provided us with accumulation data that were helpful in the interpretation of the microwave signals. We are also grateful to the three reviewers of this paper whose insightful comments and suggestions significantly improved its content.

\section{REFERENCES}

Abdalati, W. and K. Steffen. 1997. Snow melt on the Greenland ice sheet as derived from passive microwave satellite data. F. Climate, 10 (2), 165-175. Alley, R. B. 1987. Texture of polar firn for remote sensing. Ann. Glaciol., 9, 1-4. Alley, R. B., E. S. Saltzman, K. M. Cuffey and J.J. Fitzpatrick. 1990. Summertime formation of depth hoar in central Greenland. Geophys. Res. Lett., 17 (12), 2393-2396.

Benson, C. S. 1962. Stratigraphic studies in the snow and firn of the Greenland ice sheet. SIPRE Res. Rep. 70.

Bolzan, J. F. and M. Strobel. 1994. Accumulation-rate variations around Summit, Greenland. f. Glaciol., 40 (134), $56-66$.

Bromwich, D. H., F. M. Robasky, R. A. Keen and J. F. Bolzan. 1993. Modeled variations of precipitation over the Greenland ice sheet. F. Climate, 6 7), 1253-1268.

Chandrasekhar, S. 1960. Radiative transfer. New York, Dover Publications.

Chang, A. T. C., P. Gloersen, T. Schmugge, T. T. Wilheit and H.J. Zwally. 1976. Microwave emission from snow and glacier ice. J. Glaciol., 16 (74), $23-39$.

Comiso, J. C., H. J. Zwally andJ. L. Saba. 1982. Radiative transfer modeling of microwave emission and dependence on firn properties. Ann. Glaciol., $3,54-58$.

Dickinson, R. E., G. A. Meehl and W. M. Washington. 1987. Ice-albedo feedback in a CO2-doubling simulation. Climatic Change, 10 (3), 241-248.

Evans, S. 1965. Dielectric properties of ice and snow - a review. J. Glaciol., 5 (42), 773-792.

Goody, R. M. 1964. Almospheric radiation I. Theoretical basis. London, Oxford University Press.

Gow, A. J. 1969. On the rates of growth of grains and crystals in South Polar firn. 7. Glaciol., 8 83 , 241-252.

Gow, A. J. 1971. Depth-time-temperature relationships of ice crystal growth in polar glaciers. CRREL Res. Rep. 300.

Hall, D. K., A. T. C. Chang and J. L. Foster. 1986. Detection of the depthhoar layer in the snow-pack of the Arctic Coastal Plain of Alaska, U.S.A., using satellite data. f. Glaciol., 32(110), 87-94.

Hall, D. K. and 6 others. 1991. Passive microwave remote and in situ measurements of Arctic and subarctic snow cover in Alaska. Remole Sensing Environ., 38 (3), 161-172.

Henderson-Sellers, A. and P. J. Robinson. 1991. Contemporary climatology. New York, John Wiley New York, Routledge Press.

Hobbs, P. V. 1974. Ice physics. Oxford, Clarendon Press.

Hollinger, J., R. Lo, G. Poe, R. Savage and J. Pierce. 1987. Special sensor microwave/imager user's guide. Washington, DC, Naval Research Laboratory.

Jezek, K. C. and 6 others. 1991. Comparison between SMMR and SSM/I passive microwave data collected over the Antarctic ice sheet. Ohio State Univ. Byrd Polar Res. Cen. Tech. Rep. 91-03.

Mätzler, C., E. Schanda, R. Hofer and W. Good. 1980. Microwave signatures of the natural snow cover at Weissfluhjoch. In Rango, A., ed. Microwave remote sensing of snowpack properties. Washington, DC, National Aeronautics and Space Administration, 203-223. (NASA CP-2153.)

Mosley-Thompson, E. and6others. 1995. Recent increase in South Pole snow accumulation. Ann. Glaciol., 21, 131-138.

Mote, T. L. and M. R. Anderson. 1995. Variations in snowpack melt on the Greenland ice sheet based on passive-microwave measurements. J. Glaciol., $41(137), 51-60$.

Mote, T. L., M. R. Anderson, K. C. Kuivinen and C. M. Rowe. 1993. Passive microwave-derived spatial and temporal variations of summer melt on the Greenland ice sheet. Ann. Glaciol., 17, 233-238.

Ohmura, A. and N. Reeh. 1991. New precipitation and accumulation maps for Greenland. F. Glaciol., 37 (125), 140-148.

Oke, T. R. 1987. Boundary layer climates. Secondedition. London, Methuen; New York, Routledge Press. 
Shuman, C. A., R. B. Alley and S. Anandakrishnan. 1993. Characterization of a hoar-development episode using SSM/I brightness temperatures in the vicinity of the GISP2 site, Greenland. Ann. Glaciol., 17, 183-188.

Stamnes, K., S. C. Tsay, W. J. Wiscombe and K. Jayaweera. 1988. Numerically stable algorithm for discrete-ordinate method of radiative transfer in a multiple scattering and emitting layered media. Appl. Opt., 27 (12), 2502-2509.

Steffen, K., W. Abdalati and J. Stroeve. 1993. Climate sensitivity studies of the Greenland ice sheet using staellite AVHRR, SMMR, SSM/I and in situ data. Meteorol. Atmos. Phys., 51, 239-258.

Steffen, K., A. Nolin, W. Abdalati and J. White. 1995. Ice core interpretation. In Steffen, K., ed. PARCA report: Greenland science and planning meeting, 1995. Boulder, CO, University of Colorado. Cooperative Institute for Research in Environmental Sciences, 10-11.

Steffen, K., W. Abdalati and I. Sherjal. In press. Faceted crystal formation in the northeast Greenland low-accumulation region. f. Glaciol.
Sturm, M., T. C. Grenfell and D. K. Perovich. 1993. Passive microwave measurements of tundra and taiga snow covers in Alaska, U.S.A. Ann. Glaciol., 17, 125-130.

Van der Veen, C.J. and K. C. Jezek. 1993. Seasonal variations in brightness temperature for central Antarctica. Ann. Glaciol., 17, 300-306.

Zwally, H.J. 1977. Microwave emissivity and accumulation rate of polar firn. 7. Glaciol., 18 79), 195-215.

Zwally, H.J., A. C. Brenner, J. A. Major, R. A. Bindschadler and J. G. Marsh. 1989. Growth of Greenland ice sheet: measurement. Science, 246 (4937), 1587-1589.

Zwally, H.J. and S. Fiegles. 1994. Extent and duration of Antarctic surface melt. f. Glaciol., 40 (136), 463-476.

Zwally, H.J. and M. B. Giovinetto. 1995. Accumulation in Antarctica and Greenland derived from passive-microwave data: a comparison with contoured compilations. Ann. Glaciol., 21, 123-130.

MS received 22 April 1997 and accepted in revised form 3 May 1998 